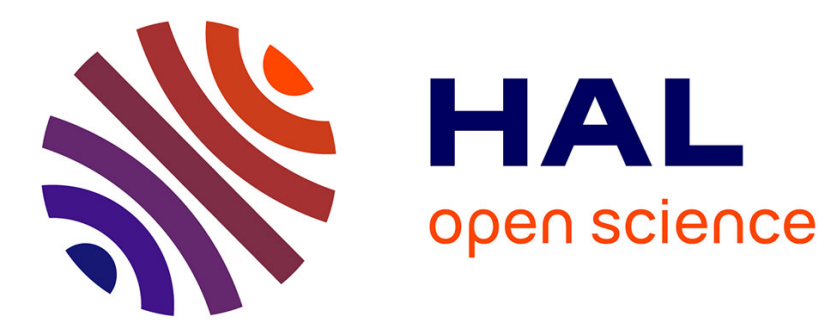

\title{
Hyperbolically frequency modulated transducer in SAW sensors and tags
}

Viktor Plesski, Marc Lamothe

\section{To cite this version:}

Viktor Plesski, Marc Lamothe. Hyperbolically frequency modulated transducer in SAW sensors and tags. Electronics Letters, 2013, 49 (24), pp.1503 - 1504. 10.1049/el.2013.2815 . hal-02300383

\section{HAL Id: hal-02300383 \\ https://hal.science/hal-02300383}

Submitted on 29 Sep 2019

HAL is a multi-disciplinary open access archive for the deposit and dissemination of scientific research documents, whether they are published or not. The documents may come from teaching and research institutions in France or abroad, or from public or private research centers.
L'archive ouverte pluridisciplinaire HAL, est destinée au dépôt et à la diffusion de documents scientifiques de niveau recherche, publiés ou non, émanant des établissements d'enseignement et de recherche français ou étrangers, des laboratoires publics ou privés. 


\section{Hyperbolically frequency modulated transducer in SAW sensors and tags}

\section{Plessky and M. Lamothe}

Linear Frequency Modulated (LFM) transducer was earlier proposed for use in SAW-tags and sensors. Here we demonstrate that the Hyperbolically Frequency Modulated (HFM) transducer and corresponding signals have significant advantages for such devices often operating in a wide range of temperatures. The HFM transducer is practically insensitive to the wide temperature variations which expand or compress signals in time. Due to exponential change of varying period with the electrode number the expansion of length of all periods is equivalent just to shift in time and the compressed signal remains practically unchanged in shape, just slightly shifted. Such shift has no importance for SAW sensors/ tags which usually operate on the difference of delays of the compressed peaks.

Introduction: The idea to use the chirp interdigital transducers (IDT) and corresponding chirp (usually LFM) signals in SAW tags and sensors was proposed some years ago [1]. It is especially attractive for the Ultra-Wide- Band (UWB) devices [2], [3], Fig.1, since the significant processing gain $B * T$ can be obtained compared to environmental parasitic reflections and thermal noise, $(B-$ being the used frequency band of the chirp signal and $T$ its duration).

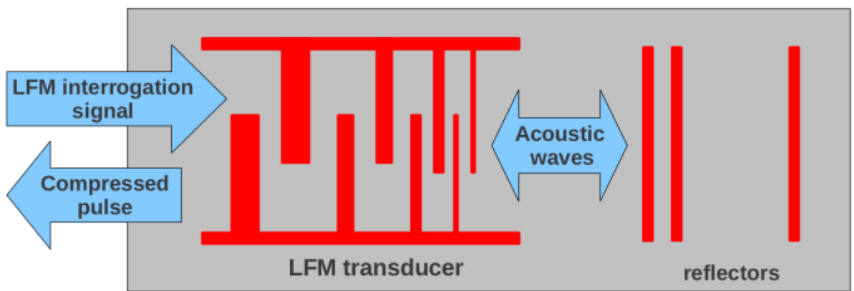

Fig. 1 SAW reflective delay line sensor with chirp transducer.

In the "orthogonal frequency coding devices"[4] the signals from different sensors are separated due to spread-spectrum technique, including IDTs or reflectors with hopping frequency. The lithium niobate $\left(\mathrm{LiNbO}_{3}\right)$ substrates often used for such devices have large temperature coefficient of delay and the increase of the delays with temperature is accompanied with decrease of frequencies, generating the change in MHz/ $\mu$ s slope of dispersion of the LFM transducer. That, finally, results in additional losses, broadening of compressed signals and/or complicated reader algorithms, especially because the sensor temperature is usually unknown in advance. The use of hyperbolically frequency modulated (HFM) signals was proposed many years ago [5] for time-invariant compression in SAW compression and expansion filters. However, the paper [5] describes only the signal properties, while design of such devices has not been disclosed. Also, for applications in radars, instead of Reflective Array Compressors, (RACs), such devices might have a visible drawback - the compressed pulse would shift with temperature. Here we demonstrate that the HFM transducer is ideal for SAW-sensor and tags applications. In such a transducer the local period of electrodes is linearly dependent on the electrode coordinate. It will be shown that it means exponential dependence of the period on the number of the electrode. The thermal variation changes all period proportionally, which for the exponential array means just a shift. Therefore such a transducer remains a kind of invariant to the temperature.

Purely geometric problem: If the period of an array increases linearly with coordinate, how can the coordinate $x_{n}$ of $n$-th element of this array be calculated? For the geometric structure of electrodes (strips, grooves, etc.) with period linearly changing with coordinate $x$ one can write the following relation:

$x_{n+1}-x_{n}=p_{0}+\varepsilon \cdot x_{n}$

Wherein $x_{n}$ is the coordinate of the centre of $n$-th electrode, $p_{0}$ - initial period, and $\varepsilon$ is the linear grows or decay (if $\varepsilon<0$ ) coefficient. Supposing that the initial electrode $(n=0)$ is situated at the origin of coordinate $0 \mathrm{X}, x_{0}=0, x_{l}=p_{0}$, the formula (1) can be treated and an equation in integer numbers, which has unique solution: $x_{n}=\frac{(1+\varepsilon)^{n}-1}{\varepsilon} \cdot p_{0}$

Or, calculating the $n$-th period:

$p_{n}=x_{n+1}-x_{n}=(1+\varepsilon)^{n} \cdot p_{0}$

Two parameters $p_{0}$ and $\varepsilon$ completely determine the array. If we fix the first period $p_{0}$ and the last period $x_{N+1^{-}} x_{N}=p_{\text {end }}$ we can find that

$\varepsilon=\left(\frac{p_{\text {end }}}{p_{0}}\right)^{\frac{1}{N}}-1$

re-write the formulas (2) and (3) in the following form:

$x_{n}=\frac{\left(\frac{p_{\text {end }}}{p_{0}}\right)^{\frac{n}{N}-1}}{\left(\frac{p_{\text {end }}}{p_{0}}\right)^{\frac{1}{N}-1}} \cdot p_{0} \quad, p_{n}=\left(\frac{p_{\text {end }}}{p_{0}}\right)^{\frac{n}{N}} \cdot p_{0}$

These formulas show that while the period of the structure changes linearly with coordinate it changes exponentially with the number of element. If the total length $L=x_{N}$ of the structure is known the following equation determines the number of periods $N$ :

$\left(\frac{p_{\text {end }}}{p_{0}}\right)^{\frac{1}{N}}=1+\frac{p_{\text {end }}-p_{0}}{L}$

Using (7) the coordinate $x_{n}$ can also be presented in the following form:

$x_{n}=L \cdot \frac{p_{0}}{p_{\text {end }}-p_{0}} \cdot\left\{\left(\frac{p_{\text {end }}}{p_{0}}\right)^{\frac{n}{N}}-1\right\}$

If our structure corresponds to a SAW propagating with velocity $V$, and the periods of the structure are related to frequency as

$p_{n}=\frac{V}{f_{n}}, \quad p_{0}=\frac{V}{\left(F_{0}-\frac{B}{2}\right)}, \quad p_{\text {end }}=\frac{V}{\left(F_{0}+\frac{B}{2}\right)}$

(here $F_{0}$ is the centre frequency, $|B|$ - the frequency band), introducing $L=x_{N}-$ total length of the structure we can represent (9) in the following form for the case when there is 1 element per period:

$x_{n}=-L \cdot \frac{F_{0}+B / 2}{B} \cdot\left\{\left(1-\frac{B \cdot V}{L \cdot\left(F_{0}^{2}-\left(\frac{B}{2}\right)^{2}\right)}\right)^{n}-1\right\}$

If in the beginning we have high frequencies, that is low period, the same formula is valid with $\mathrm{B}<0$. The same formulas can be obtained considering phase of HFM signal [5]

HFM SAW transducer: The above formula (11) is suitable for calculation of position of $90^{\circ}$ - reflectors in RAC devices, operation with HFM signals and having 1 reflector per period. For SAW transducer, we must put the series of electrodes of alternating +/polarity electrodes that is 2 per period. We can re-write formula (11) in the following equivalent form

$x_{n}=V \cdot T \cdot \frac{F_{0}+B / 2}{B} \cdot\left\{1-e^{-\frac{B}{2 T \cdot\left(F_{0}^{2}-B^{2} / 4\right)} \cdot n}\right\}$

If $B>0$ the period decreases along the structure, and if $B<0$, it is linearly increasing along the structure. The same formula could be used for $180^{\circ} \mathrm{HFM}$ reflector

Numeric simulations: As an example we simulated numerically using precise FEM/BEM software belonging to GVR Trade SA [6]. We use (Fig.2) $128^{\circ}-\mathrm{LiNbO}_{3}$ substrate $\mathbf{1}$ with HFM IDT $\mathbf{2}$ operating in frequency range $200 \mathrm{MHz}-400 \mathrm{MHz}$. The wide band transducer 3 is placed in the acoustic channel 4 of the HFM transducer allowing direct analyzing of signals generated by HFM transducer. The dispersive delay time $\boldsymbol{T}$ is equal to $0.5 \mu \mathrm{s}, \mathrm{B}^{*} \mathrm{~T}$ product thus being $\mathrm{B} * \mathrm{~T}=100$. The period of the HFM transducer at high frequency end is $9.6 \mu \mathrm{m}$, linearly increasing with distance to 2 time larger value at the low frequency end. The transducer includes 279 fingers. The wide band standard IDT including 5 electrodes with period $\lambda=12.7 \mu \mathrm{m}$ is situated in $1000 \mu \mathrm{m}$ (Marc: please check!) from HFM transducer on high frequency side of it. Both transducers have aperture $\mathrm{W}=600 \mu \mathrm{m}$.

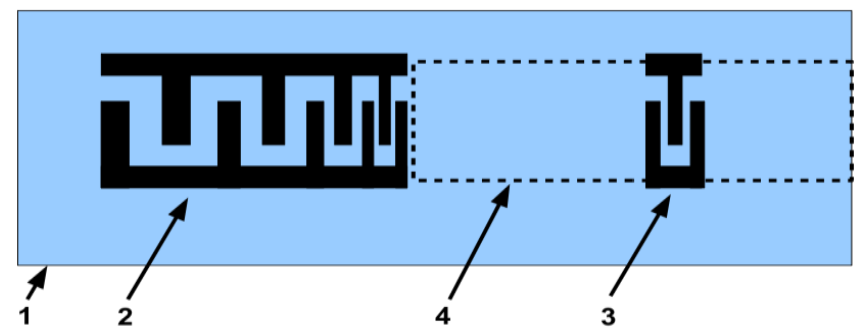

Fig. 2 Geometry used in the numeric simulation.

We simulate the temperature effect on the delay line by increasing all dimensions in the propagation direction by $2 \%$ and decreasing the 
characteristic frequencies $\left(F_{0}\right.$ and $\left.B\right)$ by $2 \%$ also. These changes roughly correspond to about $250^{\circ} \mathrm{C}$ increase of the device temperature.

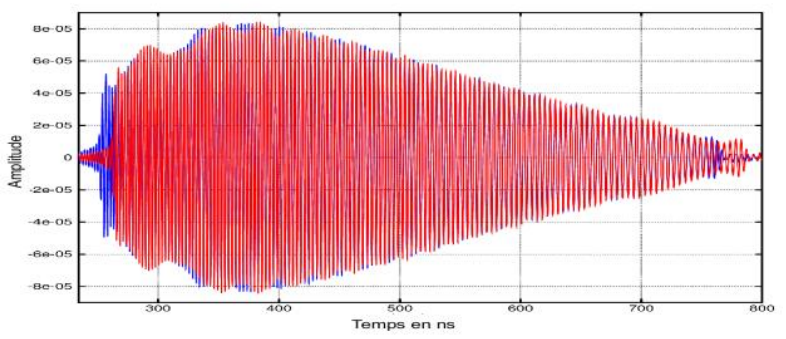

Fig. 3 Simulated impulse responses

Fig. 3 shows the impulse response of the devices. The expanded by $2 \%$ delay line has longer response. However, the main part of this response geometrically is not only similar, but identical to the initial response, when being shifted appropriately, as illustrates Fig.3. Namely this feature of HFM signal allows us using the same all "matched-tosignal" filter [7] for all expanded copies of the initial signal. We have simulated compression procedure numerically using only one, initial, signal for creation the "matched-to-signal" filter. The results are shown in Fig. 4. Both signals (initial, ideally matched to our filter, and extended by $2 \%$ ) are compressed with the same amplitude and width of the both compressed peaks is identical (close to $1 / \mathrm{B}=5 \mathrm{~ns}$ at $-3 \mathrm{~dB}$ ). In practice it means that the result is temperature-invariant: whatever is the temperature of the device its response can be compressed by the same filter.

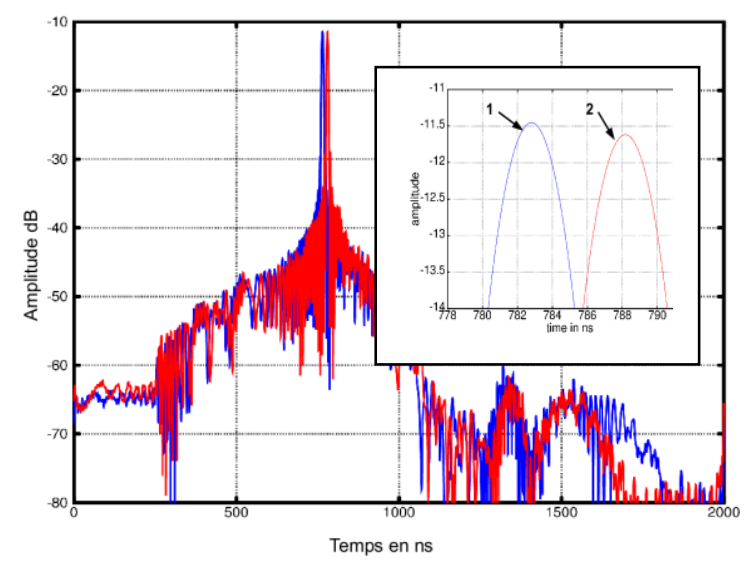

Fig. 4 Compressed HFM pulses

In SAW sensors, Fig.1, (or in SAW-tags) such common for all reflectors shift of compressed reflected pulses is not so important, since the information is extracted from distance variation between the pulses.

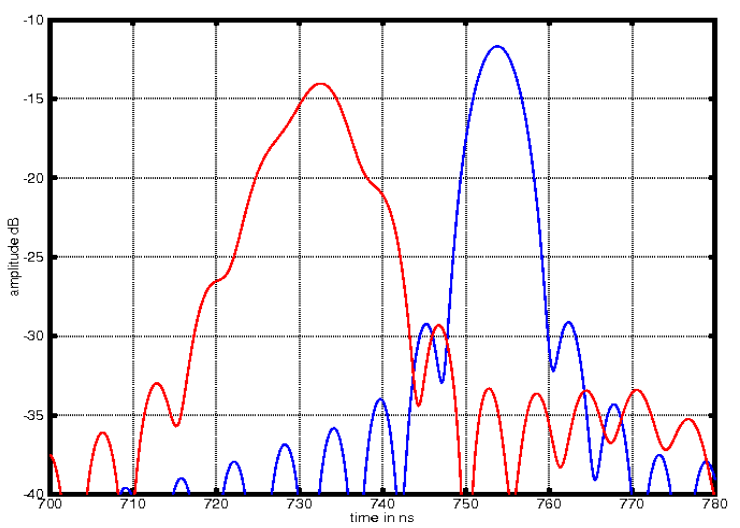

Fig. 5 Compressed pulses, LFM case. Right curve compressed pulse at initial temperature, left curve $-2 \%$ expanded chirp.

We have repeated the same numeric experiment with similar device but with LFM transducer: having the same frequency band, dispersive delay, etc. If we use inversed in time impulse response for "matched to signal" filter, the compression visibly degraded for $2 \%$ expanded LFM device. For larger $\mathrm{B} * \mathrm{~T}$ product the degradation would be even more pronounced.

Conclusion: The Hyperbolically Frequency Modulated signals and transducers (eventually reflectors) are ideally suitable for SAW-sensors and SAW-tags, since compression of such signals, being temperatureinvariant, can be achieved with always the same matched-to-signal filter, simplifying significantly the interrogation algorithm.

Acknowledgments: The authors thank S. Ballandras and J.-M. Friedt for constant support and numerous discussions

V.Plessky and M. Lamothe (GVR Trade SA, Ch.de la Rose,10, 2025, Gorgier, Switzerland),

E-mail: victor.plessky@gvrtrade.com

M. Lamothe is also with CNRS, FEMTO ST, Besançon, France.

\section{References}

1 Ianelli, Z., and Koslar, M.. : 'Surface-wave transducer device and identification system with such device', US patent, 6,788,204 B1, Sept.7, 2004

2 Harma, S., Plessky, V.P., Xianyi Li, Hartogh, P., 'Feasibility of ultra-wideband SAW RFID tags meeting FCC rules', IEEE Trans. on UFFC, 2009, 56, ( 4 ), pp. 812-820

3 Lamothe, M., and Plessky,V. : 'Temperature measurements with Ultra-Wideband SAW sensors'. Proc. IEEE Ultrasonics Symp., Dresden, Germany, Oct. 2012, pp. 2089 - 2093

4 Malocha, D.C., Puccio, D., and Gallagher, D.: 'Orthogonal frequency coding for SAW device applications', Proc. 2004 IEEE Ultrasonics Symp., pp. $1082-1085$

5 Thornton, M.J., and Rhodes, J. D.: 'Temperature-invariant pulse compression using S.A.W. devices', Microwave, Optics and Acoustics., Jan.1977, 1, (2), pp. 70-74

6 Plessky, V. P.: 'FEMSAW - an extremely accurate and powerful 2D FEM/BEM simulation tool for SAW devices',

http://www.gvrtrade.com, accessed $16^{\text {nd }}$ August 2013

7 Morgan, D.P.: 'Surface-wave devices for signal processing' (Elsevier, Amsterdam, 1985) 